\title{
SPECTRAL PROPERTIES OF PRECONDITIONED RATIONAL TOEPLITZ MATRICES*
}

\author{
TAKANG $\mathrm{KU}^{\dagger}$ AND C.-C. JAY KUO ${ }^{\dagger}$
}

\begin{abstract}
Various Toeplitz preconditioners $P_{N}$ have recently been proposed so that an $N \times N$ symmetric positive definite Toeplitz system $T_{N} \mathbf{x}=\mathbf{b}$ can be solved effectively by the preconditioned conjugate gradient (PCG) method. It has been proven that if $T_{N}$ is generated by a positive function in the Wiener class, the eigenvalues of the preconditioned matrices $P_{N}^{-1} T_{N}$ are clustered between $(1-\epsilon, 1+\epsilon)$ except for a fixed number independent of $N$. In this research, the spectra of $P_{N}^{-1} T_{N}$ are characterized more precisely for rational Toeplitz matrices $T_{N}$ with preconditioners proposed by Strang [Stud. Appl. Math., 74 (1986), pp. 171-176] and Ku and Kuo [IEEE Trans. Signal Process., 40 (1992), pp. 129-141]. The eigenvalues of $P_{N}^{-1} T_{N}$ are classified into two classes, i.e., the outliers and the clustered eigenvalues, depending on whether they converge to 1 asymptotically. It is proved that the number of outliers depends on the order of the rational generating function, and the clustering radius $\epsilon$ is proportional to the magnitude of the last element in the generating sequence used to construct these preconditioners. For the special case with $T_{N}$ generated by a geometric sequence, this approach can be used to determine the exact eigenvalue distribution of $P_{N}^{-1} T_{N}$ analytically.
\end{abstract} function

Key words. Toeplitz matrix, preconditioned conjugate gradient method, rational generating

\section{AMS(MOS) subject classifications. $65 \mathrm{~F} 10,65 \mathrm{~F} 15$}

1. Introduction. The system of linear equations associated with a symmetric positive definite (SPD) Toeplitz matrix arises in many applications, such as time series analysis and digital signal processing. The $N \times N$ symmetric Toeplitz system $T_{N} \mathbf{x}=\mathbf{b}$ is conventionally solved by algorithms based on the Levinson recursion formula [10], [16] with $O\left(N^{2}\right)$ operations. Superfast algorithms with $O\left(N \log ^{2} N\right)$ complexity have been studied intensively in the last ten years [1], [2], [3], [13]. More recently, Strang [19] proposed using an iterative method, i.e., the preconditioned conjugate gradient (PCG) method, to solve SPD Toeplitz systems and, as a consequence, the design of effective Toeplitz preconditioners has received much attention.

Strang's preconditioner $S_{N}$ [19] is obtained by preserving the central half-diagonals of $T_{N}$ and using them to form a circulant matrix. Since $S_{N}$ is circulant, the matrixvector product $S_{N}^{-1} \mathbf{v}$ can be conveniently computed via fast Fourier transform (FFT) with $O(N \log N)$ operations. It has been shown by R. Chan and Strang [5], [7] that if $T_{N}$ is generated by a positive function in the Wiener class, the eigenvalues of the preconditioned matrices $P_{N}^{-1} T_{N}$ are clustered between $(1-\epsilon, 1+\epsilon)$ except for a fixed number independent of $N$. Another preconditioner $C_{N}$ was proposed by T. Chan [8] and is defined to be the circulant matrix that minimizes the Frobenius norm $\left\|R_{N}-T_{N}\right\|_{F}$ over all circulant matrices $R_{N}$ of size $N \times N$. This turns out to be a simple optimization problem, and the elements of $C_{N}$ can be computed directly from the elements of $T_{N}$. The spectrum of $C_{N}^{-1} T_{N}$ is asymptotically equivalent to that of $S_{N}^{-1} T_{N}$ [6], and thus $C_{N}$ and $S_{N}$ have similar asymptotic behavior. In addition to preconditioners in circulant matrix form, preconditioners in skew-circulant matrix form [9] have been studied by Huckle [14]. We recently proposed a general approach

* Received by the editors September 11, 1990; accepted for publication (in revised form) March 3, 1991. This work was supported by the University of Southern California Faculty Research and Innovation Fund and by a National Science Foundation Research Initiation Award (ASC-9009323).

$\dagger$ Signal and Image Processing Institute and Department of Electrical Engineering-Systems, University of Southern California, Los Angeles, California 90089-2564 (tkku@sipi.usc.edu and cckuo@sipi.usc.edu). 
for constructing Toeplitz preconditioners [15]. Under this framework, preconditioners in circulant and skew-circulant matrix forms can be viewed as special cases and, more interestingly, preconditioners that are neither circulant nor skew-circulant can also be derived.

In [15], four new preconditioners $K_{i, N}, i=1,2,3,4$ were constructed, and it was demonstrated numerically that they have better convergence performances than other preconditioners for rational Toeplitz matrices. It was also observed in [15] that for $T_{N}$ generated by a positive rational function of order $(p, q)$ in the Wiener class, the spectra of the preconditioned matrix $P_{N}^{-1} T_{N}$ with preconditioners $S_{N}$ and $K_{i, N}, i=1,2,3,4$, have strong regularities. These regularities are stated as follows. Let the eigenvalues of $P_{N}^{-1} T_{N}$ be classified into two classes, i.e., the outliers and the clustered eigenvalues, depending on whether they converge to 1 asymptotically. Then, (1) the number of outliers is at most $2 \max (p, q)$; and (2) the clustered eigenvalues are confined in an interval $(1-\epsilon, 1+\epsilon)$ with the radius $\epsilon$ proportional to the magnitude of the last element in the generating sequence used to construct the preconditioner. The main objective of this research is to prove these two spectral properties analytically.

With the above spectral regularities, the number of iterations required to reduce the norm of the residual $\left\|\mathbf{b}-T_{N} \mathbf{x}_{k}\right\|$ by a constant factor does not increase with the problem size $N$ so that the solution of the system $T_{N} \mathbf{x}=\mathbf{b}$ can be accomplished with $\max (p, q) \times O(N \log N)$ operations. In addition, the superior performance of preconditioners $K_{i, N}$ can be easily explained by these spectral regularities. That is, for $T_{N}$ generated by a positive rational function in the Wiener class, the last elements used to construct $K_{i, N}$ and $S_{N}$ are, respectively, $t_{N}$ and $t_{[N / 2]}$ so that the corresponding radii are $\epsilon_{K}=O\left(\left|t_{N}\right|\right)$ and $\epsilon_{S}=O\left(\left|t_{\lceil N / 2\rceil}\right|\right)$. Since $O\left(\left|t_{N}\right|\right)<<O\left(\left|t_{\lceil N / 2\rceil}\right|\right)$ for sufficiently large $N$, the PCG method with preconditioners $K_{i, N}$ converges faster than with preconditioner $S_{N}$.

We should point out that the first spectral property was recently proved by Trefethen. In [23], he used the theory of CF (Carathéodory and Fejér) approximation [22] to show that $S_{N}^{-1} T_{N}$ has at most $1+2 \max (p, q)$ distinct eigenvalues asymptotically. A different approach is adopted in this paper to prove this property for both $S_{N}^{-1} T_{N}$ and $K_{i, N}^{-1} T_{N}$ (see Lemmas 2 and 8). Besides, since the first property only characterizes the spectrum of $P_{N}^{-1} T_{N}$ for infinite $N$, whereas the second property characterizes the spectrum of $P_{N}^{-1} T_{N}$ for both finite and infinite $N$, our results have a greater generality.

There exist direct methods that solve rational Toeplitz systems with $\max (p, q) \times$ $O(N)$ operations [11], [24], [25]. However, the PCG method has three advantages compared with these direct methods. First, to implement the PCG algorithm, we only need a finite segment of the generating sequence $t_{n}, n=0,1, \ldots, N-1$, which is provided by the problem, rather than the precise formula of the rational generating function. Second, the PCG method can be easily parallelized due to the parallelism provided by FFT, and it is possible to reduce the time complexity to $\max (p, q) \times O(\log N)$. In contrast, these direct methods are sequential algorithms, and the time complexity cannot be further reduced. Third, the PCG method is more widely applicable. For example, it can also be applied to Toeplitz systems with nonrational Toeplitz generating functions or those arising from the multidimensional space.

This paper is organized as follows. In $\S 2$, we briefly review the construction of preconditioners $K_{i, N}$ and summarize some of their spectral properties studied in [15]. In $\S \S 3$ and 4 , we prove the desired spectral properties of $K_{i, N}^{-1} T_{N}$ described above. The 
main idea is to transform the original generalized eigenvalue problem to an equivalent problem with nearly banded Toeplitz matrices. A similar approach is used to study the spectral properties of $S_{N}^{-1} T_{N}$, which is presented in $\S 5$. In $\S 6$, we use the analysis in $\S \S 3-5$ to determine the analytical eigenvalue distributions of $K_{i, N}^{-1} T_{N}$ and $S_{N}^{-1} T_{N}$ for Toeplitz matrices with a geometric generating sequence.

2. Construction and spectral properties of Toeplitz preconditioners $K_{i, N}, i=1,2,3,4$. Let $T_{m}$ be a sequence of $m \times m$ symmetric positive definite Toeplitz matrices with generating sequence $t_{n}$. Then,

$$
T_{N}=\left[\begin{array}{ccccc}
t_{0} & t_{1} & \cdot & t_{N-2} & t_{N-1} \\
t_{1} & t_{0} & t_{1} & \cdot & t_{N-2} \\
\cdot & t_{1} & t_{0} & \cdot & \cdot \\
t_{N-2} & \cdot & \cdot & \cdot & t_{1} \\
t_{N-1} & t_{N-2} & \cdot & t_{1} & t_{0}
\end{array}\right] .
$$

Preconditioners $K_{i, N}, i=1,2,3,4$, for $T_{N}$ are constructed by relating $T_{N}$ to a $2 N \times 2 N$ circulant matrix $R_{2 N}$,

$$
R_{2 N}=\left[\begin{array}{cc}
T_{N} & \triangle T_{N} \\
\triangle T_{N} & T_{N}
\end{array}\right]
$$

where $\triangle T_{N}$ is determined by the elements of $T_{N}$ to make $R_{2 N}$ circulant, i.e.,

$$
\Delta T_{N}=\left[\begin{array}{ccccc}
c & t_{N-1} & \cdot & t_{2} & t_{1} \\
t_{N-1} & c & t_{N-1} & \cdot & t_{2} \\
\cdot & t_{N-1} & c & \cdot & \cdot \\
t_{2} & \cdot & \cdot & \cdot & t_{N-1} \\
t_{1} & t_{2} & \cdot & t_{N-1} & c
\end{array}\right]
$$

with a constant $c$. If the behavior of the sequence $t_{n}$ is known, we choose $c$ to be $t_{N}$. Otherwise, $c=0$.

Consider the following augmented circulant system:

$$
\left[\begin{array}{cc}
T_{N} & \triangle T_{N} \\
\triangle T_{N} & T_{N}
\end{array}\right]\left[\begin{array}{l}
\mathbf{x} \\
\mathbf{x}
\end{array}\right]=\left[\begin{array}{l}
\mathbf{b} \\
\mathbf{b}
\end{array}\right]
$$

The solution of the above circulant system can be computed efficiently via FFT with $O(N \log N)$ operations. Since $(2.2)$ is equivalent to

$$
\left(T_{N}+\triangle T_{N}\right) \mathbf{x}=\mathbf{b}
$$

this implies that $\left(T_{N}+\Delta T_{N}\right)^{-1} \mathbf{b}$ can be computed efficiently and that

$$
K_{1, N}=T_{N}+\triangle T_{N}
$$

can be used as a preconditioner for $T_{N}$. Three other preconditioners can be constructed in a similar way by assuming negative, even and odd periodicities for $\mathbf{x}$ and b. We summarize the augmented systems and the corresponding preconditioners as follows:

$$
\begin{aligned}
& {\left[\begin{array}{cc}
T_{N} & \Delta T_{N} \\
\triangle T_{N} & T_{N}
\end{array}\right]\left[\begin{array}{c}
\mathbf{x} \\
-\mathbf{x}
\end{array}\right]=\left[\begin{array}{c}
\mathbf{b} \\
-\mathbf{b}
\end{array}\right] \text { and } K_{2, N}=T_{N}-\Delta T_{N}} \\
& {\left[\begin{array}{cc}
T_{N} & \Delta T_{N} \\
\triangle T_{N} & T_{N}
\end{array}\right]\left[\begin{array}{c}
\mathbf{x} \\
J_{N} \mathbf{x}
\end{array}\right]=\left[\begin{array}{c}
\mathbf{b} \\
J_{N} \mathbf{b}
\end{array}\right] \text { and } K_{3, N}=T_{N}+J_{N} \Delta T_{N}} \\
& {\left[\begin{array}{cc}
T_{N} & \Delta T_{N} \\
\triangle T_{N} & T_{N}
\end{array}\right]\left[\begin{array}{c}
\mathbf{x} \\
-J_{N} \mathbf{x}
\end{array}\right]=\left[\begin{array}{c}
\mathbf{b} \\
-J_{N} \mathbf{b}
\end{array}\right] \text { and } K_{4, N}=T_{N}-J_{N} \Delta T_{N}}
\end{aligned}
$$


where $J_{N}$ is the $N \times N$ symmetric elementary matrix which has, by definition, ones along the secondary diagonal and zeros elsewhere $\left(J_{N, i, j}=1\right.$ if $i+j=N+1$ and $J_{N, i, j}=0$ if $i+j \neq N+1$ ).

Since preconditioners $K_{i, N}, i=1,2,3,4$, correspond to $2 N$-circulant systems, the matrix-vector product $K_{i, N}^{-1} \mathbf{v}$ for an arbitrary $\mathbf{v}$ can be achieved via $2 N$-point FFT with $O(N \log N)$ operations. However, we should point out that $K_{1, N}$ is circulant and $K_{2, N}$ is skew-circulant so that $K_{1, N}^{-1} \mathbf{v}$ and $K_{2, N}^{-1} \mathbf{v}$ can be computed via $N$-point FFT. Although preconditioners $K_{3, N}$ and $K_{4, N}$ are neither circulant nor skew-circulant, $K_{3, N}^{-1} \mathbf{v}$ and $K_{4, N}^{-1} \mathbf{v}$ can be computed via $N$-point fast cosine and sine transforms, respectively. The operation count for $N$-point fast cosine (or sine) transform is approximately equal to that of $N$-point FFT in both the order and the proportional constant [17], [18], [27]. Therefore, the computational cost for the preconditioning step $K_{i, N}^{-1} \mathbf{v}$ with $i=1,2,3,4$ is about the same. For more details in implementing the PCG algorithm, we refer to [15].

To understand the relationship between the spectra of $K_{i, N}^{-1} T_{N}, i=1,2,3,4$, we rewrite the eigenvalues of $K_{i, N}^{-1} T_{N}$ as

$$
\begin{aligned}
{\left[\lambda\left(K_{i, N}^{-1} T_{N}\right)\right]^{-1} } & =\lambda\left(T_{N}^{-1}\left(T_{N}+K_{i, N}-T_{N}\right)\right)=\lambda\left(I+T_{N}^{-1}\left(K_{i, N}-T_{N}\right)\right) \\
& =1+\lambda\left(T_{N}^{-1}\left(K_{i, N}-T_{N}\right)\right),
\end{aligned}
$$

and examine the relationship between the spectra of $T_{N}^{-1}\left(K_{i, N}-T_{N}\right)$. This is characterized by the following theorem.

THEOREM 1. Let $Q_{i}$ be the set of the absolute values of the eigenvalues of $T_{N}^{-1}\left(K_{i, N}-T_{N}\right)$, i.e.,

$$
Q_{i}=\left\{|\lambda|:\left(K_{i, N}-T_{N}\right) \mathbf{x}=\lambda T_{N} \mathbf{x}\right\}, \quad i=1,2,3,4 .
$$

Then, $Q_{1}=Q_{2}=Q_{3}=Q_{4}$.

Proof. See [15] for the proof.

The above theorem can be stated alternatively as follows. Let $\lambda$ be an arbitrary eigenvalue of $T_{N}^{-1}\left(K_{i, N}-T_{N}\right)$; then there exists an eigenvalue of $T_{N}^{-1}\left(K_{j, N}-T_{N}\right)$, where $j \neq i$, with magnitude $|\lambda|$. From (2.3), spectra of $T_{N}^{-1}\left(K_{i, N}-T_{N}\right)$ clustered around zero are equivalent to those of $K_{i, N}^{-1} T_{N}$ clustered around unity. Since spectra of $T_{N}^{-1}\left(K_{i, N}-T_{N}\right)$ are clustered in a very similar pattern, so are those of $K_{i, N}^{-1} T_{N}$.

We assume that the generating sequence $t_{n}$ for the sequence of Toeplitz matrices $T_{m}$ satisfies the following two conditions:

$$
\begin{gathered}
\sum_{-\infty}^{\infty}\left|t_{n}\right|<\infty, \\
T\left(e^{i \theta}\right)=\sum_{-\infty}^{\infty} t_{n} e^{-i n \theta} \geq \delta>0 \quad \forall \theta,
\end{gathered}
$$

and the resulting matrices are said to be generated by a positive function in the Wiener class. Since $T\left(e^{i \theta}\right)$ describes the asymptotic eigenvalue distribution of $T_{m}$, the above conditions assume that the eigenvalues of $T_{m}$ are bounded and uniformly positive, asymptotically. With (2.4) and (2.5), two spectral properties of $K_{i, N}^{-1} T_{N}$ are derived. 
TheOREM 2. Preconditioners $K_{i, N}, i=1,2,3,4$, for symmetric positive definite Toeplitz matrices $T_{N}$ with the generating sequence satisfying (2.4) and (2.5) are uniformly positive definite and bounded for sufficiently large $N$.

Proof. See [15] for the proof.

THEOREM 3. Let $T_{N}$ be the $N \times N$ matrix in a sequence of $m \times m$ symmetric positive definite Toeplitz matrices $T_{m}$ with the generating sequence satisfying (2.4) and (2.5). The eigenvalues of the matrix $T_{N}^{-1}\left(K_{i, N}-T_{N}\right)$ are clustered between $(-\epsilon,+\epsilon)$ except for a finite number of outliers for sufficiently large $N(\epsilon)$.

Proof. See [15] for the proof.

Theorems 2 and 3 hold for both rational and nonrational Toeplitz matrices satisfying (2.4) and (2.5). However, when $T_{N}$ is additionally rational, we are able to obtain stronger results and characterize the spectra of $K_{i, N}^{-1} T_{N}$ more precisely. In $\S \S 3$ and 4 , we focus on the spectrum of $K_{1, N}^{-1} T_{N}$, from which the spectra of $K_{i, N}^{-1} T_{N}, i=2,3,4$, can be estimated based on Theorem 1 .

3. Rational generating functions for $\triangle T_{N}$. Due to (2.3), the spectral properties of $K_{1, N}^{-1} T_{N}$ can be determined by examining those of $T_{N}^{-1} \triangle T_{N}$, where $\triangle T_{N}$ is given in (2.1) with $c=t_{N}$. Let $t_{n},-\infty<n<\infty$, be the generating sequence of a sequence of $m \times m$ Toeplitz matrices $T_{m}$. The Laurent series

$$
T(z)=\sum_{n=-\infty}^{\infty} t_{n} z^{-n}
$$

is known as the generating function of these matrices. If matrices $T_{m}$ are symmetric, we decompose $T(z)$ into

$$
T(z)=T_{+}\left(z^{-1}\right)+T_{+}(z)
$$

where

$$
T_{+}\left(z^{-1}\right)=\frac{t_{0}}{2}+\sum_{n=1}^{\infty} t_{n} z^{-n} .
$$

Thus $T(z)$ is completely characterized by $T_{+}\left(z^{-1}\right)$. Additionally, if

$$
T_{+}\left(z^{-1}\right)=\frac{A\left(z^{-1}\right)}{B\left(z^{-1}\right)}=\frac{a_{0}+a_{1} z^{-1}+\cdots+a_{p} z^{-p}}{b_{0}+b_{1} z^{-1}+\cdots+b_{q} z^{-q}},
$$

where $b_{0}=1, a_{p} b_{q} \neq 0$, and polynomials $A\left(z^{-1}\right)$ and $B\left(z^{-1}\right)$ have no common factor, we call $T_{m}$ the rational Toeplitz matrices generated by a rational function of order $(p, q)$. From (3.1) and (3.3), we have

$$
T(z)=\frac{A\left(z^{-1}\right)}{B\left(z^{-1}\right)}+\frac{A(z)}{B(z)} .
$$

It is well known [12] that there exists an isomorphism between the ring of the power series $P\left(z^{-1}\right)=\sum_{n=0}^{\infty} p_{n} z^{-n}$ (or $\left.P(z)\right)$ and the ring of the semi-infinite lower (or upper) triangular Toeplitz matrices with $p_{0}, p_{1}, \cdots, p_{n}, \cdots$ as the first column (or row). The power series multiplication is isomorphic to matrix multiplication. By applying the isomorphism to (3.4) and focusing on the leading $N \times N$ blocks of the corresponding matrices, we derive the following relationship [12]:

$$
T_{N}=L_{a} L_{b}^{-1}+U_{a} U_{b}^{-1}
$$


where $L_{a}$ (or $U_{a}$ ) is an $N \times N$ lower (or upper) triangular Toeplitz matrix with first $N$ coefficients in $A\left(z^{-1}\right)$ as its first column (or row). Matrices $L_{b}$ and $U_{b}$ are defined similarly with respect to $B\left(z^{-1}\right)$. We can also establish an expression similar to (3.5) for $\Delta T_{N}$. To do so, we first note that the sequence $t_{n}$ is recursively defined for large $n$. This is stated as follows.

LEMMA 1. The sequence $t_{n}$ generated by (3.2) and (3.3) follows the recursion,

$$
t_{n+1}=-\left(b_{1} t_{n}+b_{2} t_{n-1}+\cdots+b_{q} t_{n-q+1}\right), \quad n \geq \max (p, q) .
$$

Proof. From (3.2) and (3.3), we have

$$
\left(\frac{t_{0}}{2}+\sum_{n=1}^{\infty} t_{n} z^{-n}\right)\left(b_{0}+b_{1} z^{-1}+\cdots+b_{q} z^{-q}\right)=a_{0}+a_{1} z^{-1}+\cdots+a_{p} z^{-p} .
$$

The proof is completed by comparing the coefficients of the above equation.

With Lemma 1, the number of outliers of $T_{N}^{-1} \triangle T_{N}$ is determined by the following lemma.

Lemma 2. Let $T_{N}$ be an $N \times N$ symmetric Toeplitz matrix generated by $T(z)$ with $T_{+}\left(z^{-1}\right)$ given by (3.3), and the corresponding generating sequence satisfies (2.4) and (2.5). $T_{N}^{-1} \triangle T_{N}$ has asymptotically at most $2 \max (p, q)$ nonzero eigenvalues (outliers).

Proof. Let us define a matrix

$$
\triangle E_{N}=\triangle F_{N}+\triangle F_{N}^{T}
$$

where

$$
\Delta F_{N}=\left[\begin{array}{ccccc}
t_{N} & t_{N-1} & \cdot & t_{2} & t_{1} \\
t_{N+1} & t_{N} & t_{N-1} & \cdot & t_{2} \\
\cdot & t_{N+1} & t_{N} & \cdot & \cdot \\
t_{2 N-2} & \cdot & \cdot & \cdot & t_{N-1} \\
t_{2 N-1} & t_{2 N-2} & \cdot & t_{N+1} & t_{N}
\end{array}\right]
$$

Since elements $t_{n}$ in $\triangle F_{N}$ satisfy (3.6), there are at most $\max (p, q)$ independent rows in $\triangle F_{N}$ and therefore, the rank of $\triangle E_{N}$ is at most $2 \max (p, q)$.

Let $\triangle P_{N}=\triangle E_{N}-\triangle T_{N} ;$ it is easy to verify that the $l_{1}$ and $l_{\infty}$ norms of $\triangle P_{N}$ are both less than

$$
\tau_{K}=2 \sum_{n=N}^{2 N-1}\left|t_{n}\right|
$$

Consequently, we have

$$
\left\|\triangle P_{N}\right\|_{2} \leq\left(\left\|\triangle P_{N}\right\|_{1}\left\|\triangle P_{N}\right\|_{\infty}\right)^{1 / 2} \leq \tau_{K}
$$

Since $\tau_{K}$ goes to zero as $N$ goes to infinity due to (2.4), and since the eigenvalues of $T_{N}^{-1}$ are bounded due to (2.5), the spectra of $T_{N}^{-1} \triangle T_{N}$ and $T_{N}^{-1} \triangle E_{N}$ are asymptotically equivalent. It follows that both $T_{N}^{-1} \triangle E_{N}$ and $T_{N}^{-1} \triangle T_{N}$ have at most $2 \max (p, q)$ nonzero eigenvalues asymptotically.

As a consequence of Lemma $2, T_{N}^{-1} \triangle T_{N}$ has at least $N-2 \max (p, q)$ eigenvalues converging to zero as the problem size $N$ becomes large. For the rest of this section and in $\S 4$, we study the clustering property of these eigenvalues. Our approach is outlined 
as follows. First, we associate $\triangle T_{N}$ with some appropriate rational generating function $\tilde{T}(z)=\tilde{T}_{+}\left(z^{-1}\right)+\tilde{T}_{+}(z)$. The forms of $\tilde{T}_{+}\left(z^{-1}\right)$ for $p \leq q$ and $p>q$ are given in Lemmas 3 and 4 , respectively. We then transform the generalized eigenvalue problem involving $T_{N}^{-1} \triangle T_{N}$ into another generalized eigenvalue problem involving $Q_{N}^{-1} \triangle Q_{N}$. We show that $Q_{N}$ and $\triangle Q_{N}$ are nearly banded Toeplitz matrices in Lemma 5 and examine the spectral property of $Q_{N}^{-1} \triangle Q_{N}$ in Lemma 6.

Since $T_{N}$ is a symmetric rational Toeplitz matrix, and the elements of $\triangle T_{N}$ are those of $T_{N}$ with reverse ordering, it is not surprising that $\triangle T_{N}$ is also generated by a certain rational function, which is determined below. Let us use the elements $t_{n}$ of a given $T_{N}$ with $N>\max (p, q)$ to construct a new sequence $\tilde{t}_{n}$. The cases $p \leq q$ and $p>q$ are considered separately.

Case 1. $p \leq q$. We choose

$$
\tilde{t}_{n}= \begin{cases}t_{N-n}, & 0 \leq n \leq q-1, \\ -\left(\sum_{k=1}^{q} b_{q-k} \tilde{t}_{n-k}\right) / b_{q}, & q \leq n .\end{cases}
$$

Note that elements $\tilde{t}_{n}$ above with $n \geq q$ are obtained based on the recursion (3.6) examined from the reverse direction.

Case 2. $p>q$. We decompose $T_{+}\left(z^{-1}\right)$ into

$$
T_{+}\left(z^{-1}\right)=F_{+}\left(z^{-1}\right)+T_{1,+}\left(z^{-1}\right)
$$

where

$$
F_{+}\left(z^{-1}\right)=f_{0}+f_{1} z^{-1}+\cdots+f_{p-q} z^{-(p-q)}
$$

and

$$
T_{1,+}\left(z^{-1}\right)=\frac{A^{\prime}\left(z^{-1}\right)}{B\left(z^{-1}\right)}=\frac{a_{0}^{\prime}+a_{1}^{\prime} z^{-1}+\cdots+a_{s}^{\prime} z^{-s}}{b_{0}+b_{1} z^{-1}+\cdots+b_{q} z^{-q}}
$$

with $s<q$. Let $t_{1, n}$ be the generating sequence of $T_{1,+}\left(z^{-1}\right)$. There exists a simple relationship between the elements of generating sequences for $T_{+}\left(z^{-1}\right)$ and $T_{1,+}\left(z^{-1}\right)$, i.e.,

$$
t_{n}= \begin{cases}t_{1, n}+f_{n}, & 0 \leq n \leq p-q \\ t_{1, n}, & p-q<n\end{cases}
$$

With respect to $T_{1,+}\left(z^{-1}\right)$ and $F_{+}\left(z^{-1}\right)$, we choose the corresponding $\tilde{t}_{1, n}$ and $\tilde{t}_{2, n}$, respectively, as

$$
\tilde{t}_{1, n}= \begin{cases}t_{1, N-n}, & 0 \leq n \leq q-1 \\ -\left(\sum_{k=1}^{q} b_{q-k} \tilde{t}_{1, n-k}\right) / b_{q}, & q \leq n\end{cases}
$$

and

$$
\tilde{t}_{2, n}= \begin{cases}f_{N-n}, & N-p+q \leq n \leq N \\ 0, & \text { elsewhere. }\end{cases}
$$

Finally, we define

$$
\tilde{t}_{n}=\tilde{t}_{1, n}+\tilde{t}_{2, n}
$$


We associate the sequence $\tilde{t}_{n}$ given by (3.7) or (3.9) with a sequence of symmetric Toeplitz matrices $\tilde{T}_{m}$. It is straightforward to verify that for $N>\max (p, q), \tilde{T}_{N}=$ $\triangle T_{N}$. The generating function for matrices $\tilde{T}_{m}$ is

$$
\tilde{T}(z)=\tilde{T}_{+}\left(z^{-1}\right)+\tilde{T}_{+}(z), \quad \text { where } \tilde{T}_{+}\left(z^{-1}\right)=\frac{\tilde{t}_{0}}{2}+\sum_{n=1}^{\infty} \tilde{t}_{n} z^{-n} .
$$

The forms of $\tilde{T}_{+}\left(z^{-1}\right)$ with $p \leq q$ and $p>q$ are described, respectively, in Lemmas 3 and 4 .

LEMMA 3. If $T_{N}$ is generated by $T(z)$ with $T_{+}\left(z^{-1}\right)$ given by (3.3), and $p \leq q<$ $N$, then $\triangle T_{N}$ is generated by $\tilde{T}(z)$ with

$$
\tilde{T}_{+}\left(z^{-1}\right)=\frac{C\left(z^{-1}\right)}{D\left(z^{-1}\right)}=\frac{c_{0}+c_{1} z^{-1}+\cdots+c_{q} z^{-q}}{d_{0}+d_{1} z^{-1}+\cdots+d_{q} z^{-q}}
$$

where

$$
d_{i}=\left\{\begin{array}{ll}
b_{q}^{-1} b_{q-i}, & 0 \leq i \leq q, \\
0, & q<i,
\end{array} \quad c_{i}= \begin{cases}\sum_{j=0}^{i} d_{j} \tilde{t}_{i-j}^{\prime}, & 0 \leq i \leq q, \\
0, & q<i,\end{cases}\right.
$$

and where

$$
\tilde{t}_{n}^{\prime}= \begin{cases}\tilde{t}_{n}, & n \neq 0 \\ \frac{\tilde{t}_{0}}{2}, & n=0\end{cases}
$$

with $\tilde{t}_{n}$ given by $(3.7)$.

Proof. By (3.7), the sequence $\tilde{t}_{n}$ satisfies the recursion

$$
\tilde{t}_{k}=-\left(d_{1} \tilde{t}_{k-1}+d_{2} \tilde{t}_{k-2}+\cdots+d_{q} \tilde{t}_{k-q}\right), \quad \text { for } k \geq q
$$

with $d_{i}$ given by (3.10a). Let us define $G_{k}\left(z^{-k}\right), k>q$, as

$$
G_{k}\left(z^{-k}\right)=\left(\tilde{t}_{k}+d_{1} \tilde{t}_{k-1}+d_{2} \tilde{t}_{k-2}+\cdots+d_{q} \tilde{t}_{k-q}\right) z^{-k}
$$

It is evident from (3.11) that $G_{k}\left(z^{-k}\right)=0$ for $k>q$. Therefore, we have

$$
\begin{aligned}
\left(1+d_{1} z^{-1}+\cdots+d_{q} z^{-q}\right) \tilde{T}_{+}\left(z^{-1}\right) & =\sum_{i=0}^{q}\left(\sum_{j=0}^{i} d_{j} \tilde{t}_{i-j}^{\prime}\right) z^{-i}+\sum_{k=q+1}^{\infty} G_{k}\left(z^{-k}\right) \\
& =c_{0}+c_{1} z^{-1}+\cdots+c_{q} z^{-q}
\end{aligned}
$$

with $c_{i}$ and $\tilde{t}_{i}^{\prime}$ defined in $(3.10 \mathrm{a})$ and $(3.10 \mathrm{~b})$, respectively. This completes the proof.

LEMMA 4. If $T_{N}$ is generated by $T(z)$ with $T_{+}\left(z^{-1}\right)$ given by (3.3), and $q<p<$ $N$, then $\triangle T_{N}$ is generated by $\tilde{T}(z)$ with

$$
\tilde{T}_{+}\left(z^{-1}\right)=\frac{C\left(z^{-1}\right)}{D\left(z^{-1}\right)}+F_{+}(z) z^{-N}
$$

where

$$
d_{i}=\left\{\begin{array}{ll}
b_{q}^{-1} b_{q-i}, & 0 \leq i \leq q, \\
0, & q<i,
\end{array} \quad c_{i}= \begin{cases}\sum_{j=0}^{i} d_{j} \tilde{t}_{i-j}^{\prime}, & 0 \leq i \leq q \\
0, & q<i\end{cases}\right.
$$


and where

$$
\tilde{t}_{n}^{\prime}=\left\{\begin{array}{cc}
\tilde{t}_{n}, & n \neq 0 \\
\frac{\tilde{t}_{0}}{2}, & n=0
\end{array}\right.
$$

with $\tilde{t}_{n}$ given by $(3.9)$.

Proof. Due to (3.8), we express $\tilde{T}_{+}\left(z^{-1}\right)$ as $\tilde{T}_{+}\left(z^{-1}\right)=\tilde{F}_{+}\left(z^{-1}\right)+\tilde{T}_{1,+}\left(z^{-1}\right)$, where

$$
\tilde{F}_{+}\left(z^{-1}\right)=\sum_{n=N-p+q}^{N} f_{N-n} z^{-n}, \quad \tilde{T}_{1,+}\left(z^{-1}\right)=\frac{\tilde{t}_{1,0}}{2}+\sum_{n=1}^{\infty} \tilde{t}_{1, n} z^{-n} .
$$

It is clear from Lemma 3 and (3.8a) that

$$
\tilde{F}_{+}\left(z^{-1}\right)=F_{+}(z) z^{-N}, \quad \tilde{T}_{1,+}\left(z^{-1}\right)=\frac{C\left(z^{-1}\right)}{D\left(z^{-1}\right)} .
$$

Thus the proof is completed.

We rewrite (3.12) as

$$
\tilde{T}_{+}\left(z^{-1}\right)=\frac{C_{1}\left(z^{-1}\right)}{D\left(z^{-1}\right)}, \quad \text { with } C_{1}\left(z^{-1}\right)=C\left(z^{-1}\right)+D\left(z^{-1}\right) F_{+}(z) z^{-N}
$$

Applying the isomorphism to (3.10) or (3.13) and focusing on the leading $N \times N$ blocks of the corresponding matrices, we obtain

$$
\tilde{T}_{N}=L_{c} L_{d}^{-1}+U_{c} U_{d}^{-1}
$$

where $L_{c}$ (or $U_{c}$ ) is an $N \times N$ lower (or upper) triangular Toeplitz matrix with the first $N$ coefficients of $C\left(z^{-1}\right)(p \leq q)$ or $C_{1}\left(z^{-1}\right)(p>q)$ as its first column (or row). Matrices $L_{d}$ and $U_{d}$ are similarly defined with respect to $D\left(z^{-1}\right)$. Since $\triangle T_{N}=\tilde{T}_{N}$, we obtain

$$
\triangle T_{N}=L_{c} L_{d}^{-1}+U_{c} U_{d}^{-1}
$$

4. Spectral properties of $T_{N}^{-1} \Delta T_{N}$. With the results given by (3.5) and (3.14), we then transform the generalized eigenvalue problem,

$$
\triangle T_{N} \mathbf{x}=\lambda T_{N} \mathbf{x}
$$

to an equivalent generalized eigenvalue problem,

$$
\triangle Q_{N} \mathbf{y}=\lambda Q_{N} \mathbf{y}
$$

where

$$
Q_{N}=L_{b} T_{N} U_{b}=L_{a} U_{b}+L_{b} U_{a}
$$

and

$$
\triangle Q_{N}=L_{b} \triangle T_{N} U_{b}=L_{b} L_{c} L_{d}^{-1} U_{b}+L_{b} U_{c} U_{d}^{-1} U_{b}
$$

It is clear that (4.1) and (4.2) have identical eigenvalues and their eigenvectors are related via $\mathbf{x}=U_{b} \mathbf{y}$. The reason for (4.2) is that $Q_{N}$ and $\triangle Q_{N}$ are nearly banded 
Toeplitz matrices which can be more easily analyzed. The properties of matrices $Q_{N}$ and $\triangle Q_{N}$ are characterized below.

LEMMA 5. Let $T_{m}$ be a sequence of $m \times m$ symmetric Toeplitz matrices generated by $T(z)$ with $T_{+}\left(z^{-1}\right)$ given by (3.3), and the corresponding generating sequence satisfies (2.4) and (2.5). The southeast $(N-\max (p, q)) \times(N-\max (p, q))$ blocks of $Q_{N}$ and $\triangle Q_{N}$ are symmetric banded Toeplitz matrices with generating functions

$$
Q(z)=A\left(z^{-1}\right) B(z)+B\left(z^{-1}\right) A(z)
$$

and

$$
\triangle Q(z)=b_{q} z^{q} C\left(z^{-1}\right) B\left(z^{-1}\right)+b_{q} z^{-q} C(z) B(z)
$$

respectively.

Proof. Consider two Toeplitz matrices $F_{N}$ and $G_{N}$ of size $N \times N$, where $F_{N}$ is a lower triangular Toeplitz matrix with lower bandwidth $r$ and the generating function $F\left(z^{-1}\right), G_{N}$ an upper triangular Toeplitz matrix with upper bandwidth $s$ and the generating function $G(z)$. It is easy to verify that the product $F_{N} G_{N}$, except for its northwest $r \times s$ block, is a banded Toeplitz matrix with the lower bandwidth $r$, upper bandwidth $s$, and generating function $F\left(z^{-1}\right) G(z)$. We generalize the above result to $Q_{N}=L_{a} U_{b}+L_{b} U_{a}$ and find that the southeast $(N-\max (p, q)) \times(N-\max (p, q))$ block of $Q_{N}$ is a symmetric banded Toeplitz matrix with the generating function

$$
Q(z)=A\left(z^{-1}\right) B(z)+B\left(z^{-1}\right) A(z) .
$$

Since the product of lower (or upper) triangular Toeplitz matrices is commutative, we rewrite $(4.2 \mathrm{~b})$ as

$$
\triangle Q_{N}=\triangle Q_{1, N}+\triangle Q_{1, N}^{T}, \quad \text { where } \triangle Q_{1, N}=L_{b} L_{c} L_{d}^{-1} U_{b} .
$$

When $p \leq q$, the product $L_{b} L_{c} L_{d}^{-1}$ results in a lower triangular Toeplitz matrix with the generating function $B\left(z^{-1}\right) C\left(z^{-1}\right) D^{-1}\left(z^{-1}\right)$. The matrix $\triangle Q_{1, N}$, except for the first $q$ columns, is a Toeplitz matrix with the generating function

$$
\triangle Q_{1, N}\left(z^{-1}\right)=B\left(z^{-1}\right) C\left(z^{-1}\right) D^{-1}\left(z^{-1}\right) B(z) .
$$

We use (3.10a) to relate $D\left(z^{-1}\right)$ with $B(z)$, i.e.,

$$
D\left(z^{-1}\right)=\sum_{n=0}^{q} d_{n} z^{-n}=b_{q}^{-1} z^{-q} \sum_{n=0}^{q} b_{q-n} z^{q-n}=b_{q}^{-1} z^{-q} B(z) .
$$

Thus $\triangle Q_{1, N}\left(z^{-1}\right)=b_{q} z^{q} B\left(z^{-1}\right) C\left(z^{-1}\right)$. Similarly, $\triangle Q_{1, N}^{T}$, except for the first $q$ rows, is a Toeplitz matrix with the generating function $\triangle Q_{1, N}(z)$. Therefore, the southeast $(N-q) \times(N-q)$ block of $\triangle Q_{N}$ is a symmetric banded Toeplitz matrix with the generating function

$$
\triangle Q(z)=\triangle Q_{1, N}\left(z^{-1}\right)+\triangle Q_{1, N}(z)=b_{q}\left(z^{q} B\left(z^{-1}\right) C\left(z^{-1}\right)+z^{-q} B(z) C(z)\right),
$$

where the coefficients of $C\left(z^{-1}\right)$ are given in Lemma 3 .

When $p>q$, the generating function of matrix $L_{c}$ is $C_{1}\left(z^{-1}\right)$ in (3.13). Consequently, $\triangle Q_{1, N}$, except for the first $q$ columns, is a Toeplitz matrix with the generating function

$$
\begin{aligned}
\triangle Q_{1, N}\left(z^{-1}\right) & =B\left(z^{-1}\right) C_{1}\left(z^{-1}\right) D^{-1}\left(z^{-1}\right) B(z) \\
& =B\left(z^{-1}\right) C\left(z^{-1}\right) D^{-1}\left(z^{-1}\right) B(z)+z^{-N} B\left(z^{-1}\right) F_{+}(z) B(z) .
\end{aligned}
$$


Recall that the orders of polynomials $B(z)$ and $F_{+}(z)$ are $q$ and $p-q$, respectively. The lowest order in $z$ of the polynomial $z^{-N} B\left(z^{-1}\right) F_{+}(z) B(z)$ is $-(N-p)$, and the elements of the leading $N \times N$ Toeplitz matrix generated by $z^{-N} B\left(z^{-1}\right) F_{+}(z) B(z)$ are zeros except for the southwest $p$ diagonals. Therefore, the matrix $\triangle Q_{1, N}$, except for the first $q$ columns and the southwest $p$ diagonals, is a Toeplitz matrix with the generating function

$$
\triangle Q_{1, N}\left(z^{-1}\right)=B\left(z^{-1}\right) C\left(z^{-1}\right) D^{-1}\left(z^{-1}\right) B(z) .
$$

Then it follows that the southeast $(N-p) \times(N-p)$ block of $\triangle Q_{N}$ is a symmetric banded Toeplitz matrix with the generating function

$$
\triangle Q(z)=b_{q}\left[z^{q} B\left(z^{-1}\right) C\left(z^{-1}\right)+z^{-q} B(z) C(z)\right]
$$

where the coefficients of $C\left(z^{-1}\right)$ are given in Lemma 4. The proof is completed.

The following lemma gives the bound of the clustered eigenvalues of $Q_{N}^{-1} \triangle Q_{N}$.

LEMMA 6. Let $T_{m}$ be a sequence of $m \times m$ symmetric Toeplitz matrices generated by $T(z)$ with $T_{+}\left(z^{-1}\right)$ given by (3.3), and let the corresponding generating sequence satisfy (2.4) and (2.5). Then $Q_{N}^{-1} \triangle Q_{N}$ has at least $N-2 \max (p, q)$ eigenvalues with magnitude bounded by

$$
\epsilon=\max _{z=e^{-i 2 \pi n / N}}\left|\frac{\triangle Q(z)}{Q(z)}\right| .
$$

Proof. Let us denote the southeast $(N-\max (p, q)) \times(N-\max (p, q))$ blocks of $Q_{N}$ and $\triangle Q_{N}$ by $\mathcal{Q}_{N-\max (p, q)}$ and $\triangle \mathcal{Q}_{N-\max (p, q)}$, respectively. By the minimax theorem (or Courant-Fisher theorem) of eigenvalues [20], [26], there are at least $N-2 \max (p, q)$ eigenvalues of $Q_{N}^{-1} \triangle Q_{N}$ bounded by the maximum and the minimum eigenvalues of $\mathcal{Q}_{N-\max (p, q)}^{-1} \triangle \mathcal{Q}_{N-\max (p, q)}$.

It is clear from Lemma 5 that $\mathcal{Q}_{N-\max (p, q)}$ and $\Delta \mathcal{Q}_{N-\max (p, q)}$ are symmetric banded Toeplitz matrices with bandwidth $\leq \max (p, q)$. We construct two $N \times N$ symmetric circulant matrices $\mathcal{R}_{N}$ and $\triangle \mathcal{R}_{N}$ with $\mathcal{Q}_{N-\max (p, q)}$ and $\triangle \mathcal{Q}_{N-\max (p, q)}$ as their leading principal submatrices, respectively. By the separation theorem (or intertwining theorem) of eigenvalues [20], [26], the eigenvalues of $\mathcal{Q}_{N-\max (p, q)}^{-1} \Delta \mathcal{Q}_{N-\max (p, q)}$ are bounded by the maximum and the minimum eigenvalues of $\mathcal{R}_{N}^{-1} \triangle \mathcal{R}_{N}$. It is well known that the eigenvalues of $\mathcal{R}_{N}^{-1} \triangle \mathcal{R}_{N}$ are

$$
\triangle Q\left(e^{-i 2 \pi n / N}\right) / Q\left(e^{-i 2 \pi n / N}\right), \quad n=0,1, \cdots, N-1 .
$$

Thus the proof is completed.

We then focus on the bound of (4.5). By using (3.1) and (3.3), $\triangle Q(z) / Q(z)$ can be further simplified as

$$
\begin{aligned}
\triangle Q(z) / Q(z) & =\left[b_{q} z^{q} B\left(z^{-1}\right) C\left(z^{-1}\right)+b_{q} z^{-q} B(z) C(z)\right] /\left[B\left(z^{-1}\right) B(z) T(z)\right] \\
& =\left[b_{q} z^{q} C\left(z^{-1}\right)\right] /[B(z) T(z)]+\left[b_{q} z^{-q} C(z)\right] /\left[B\left(z^{-1}\right) T(z)\right] .
\end{aligned}
$$

Since $T\left(e^{i \theta}\right)=A\left(e^{-i \theta}\right) / B\left(e^{-i \theta}\right)+A\left(e^{i \theta}\right) / B\left(e^{i \theta}\right)$, and $\left|T\left(e^{i \theta}\right)\right|$ is finite from (2.4), $\left|B\left(e^{i \theta}\right)\right|$ is uniformly positive, i.e.,

$$
\left|B\left(e^{i \theta}\right)\right| \geq \beta>0 .
$$


Combining (2.5), (4.6), and (4.7), we obtain

$$
\left|\frac{\triangle Q\left(e^{-i \theta}\right)}{Q\left(e^{-i \theta}\right)}\right| \leq\left|\frac{2 b_{q} C\left(e^{-i \theta}\right)}{\beta \delta}\right|
$$

with arbitrary $\theta$.

We then focus our discussion on the bound of $\left|b_{q} C\left(e^{-i \theta}\right)\right|$. First, we have

$$
\left|b_{q} C\left(e^{-i \theta}\right)\right| \leq \sum_{i=0}^{q}\left|b_{q} c_{i}\right|=\sum_{i=0}^{q}\left|\sum_{j=0}^{i} b_{q} d_{j} \tilde{t}_{i-j}^{\prime}\right|=\sum_{i=0}^{q}\left|\sum_{j=0}^{i} b_{q-j} t_{N-i+j}\right|,
$$

where the last equality is due to (3.7), (3.10a), and (3.10b). Since $t_{n}$ satisfies the recursion (3.6), we use the equality

$$
\sum_{j=0}^{q} b_{q-j} t_{N-i+j}=0
$$

with $N>\max (p, q)$ to simplify (4.9), i.e.,

$$
\begin{aligned}
\left|b_{q} C\left(e^{-i \theta}\right)\right| & \leq \sum_{i=0}^{q}\left|-\sum_{j=i+1}^{q} b_{q-j} t_{N-i+j}\right| \leq \sum_{i=0}^{q} \sum_{j=i+1}^{q}\left|b_{q-j}\right|\left|t_{N-i+j}\right| \\
& \leq \max _{N \leq n \leq N+q}\left|t_{n}\right| \sum_{i=0}^{q} \sum_{j=i+1}^{q}\left|b_{q-j}\right| .
\end{aligned}
$$

Furthermore, the term $\sum_{i=0}^{q} \sum_{j=i+1}^{q}\left|b_{q-j}\right|$ is bounded by

$$
\sum_{i=0}^{q} \sum_{j=i+1}^{q}\left|b_{q-j}\right|<\sum_{i=0}^{q} \sum_{j=1}^{q}\left|b_{q-j}\right|<(q+1) \sum_{j=0}^{q}\left|b_{j}\right|<(q+1) 2^{q}
$$

where the last inequality is due to the following lemma.

LEMMA 7. Let $T_{m}$ be a sequence of $m \times m$ symmetric Toeplitz matrices generated by $T(z)$ with $T_{+}\left(z^{-1}\right)$ given by (3.3), and let the corresponding generating sequence satisfy (2.4) and (2.5); then

$$
\sum_{j=0}^{q}\left|b_{j}\right|<2^{q}
$$

Proof. Since $B\left(z^{-1}\right)$ is a polynomial in $z$ of order $q, B\left(z^{-1}\right)$ can be factorized as

$$
B\left(z^{-1}\right)=\sum_{i=0}^{q} b_{i} z^{-i}=\left(1-r_{1} z^{-1}\right)\left(1-r_{2} z^{-1}\right) \cdots\left(1-r_{q} z^{-1}\right)
$$

where $r_{i}, 1 \leq i \leq q$, are poles of $T_{+}\left(z^{-1}\right)$. A direct consequence of (2.4) is that all poles of $T_{+}\left(z^{-1}\right)$ should lie inside the unit circle, i.e., $\left|r_{i}\right|<1,1 \leq i \leq q$. It is clear from (4.12) that

$$
\left|b_{k}\right| \leq\left(\begin{array}{c}
q \\
k
\end{array}\right)\left(\max \left|r_{i}\right|\right)^{k}<\left(\begin{array}{c}
q \\
k
\end{array}\right), \quad \text { where }\left(\begin{array}{c}
q \\
k
\end{array}\right) \equiv \frac{q !}{(q-k) ! k !}
$$


Therefore, we have

$$
\sum_{j=0}^{q}\left|b_{j}\right|<\sum_{j=0}^{q}\left(\begin{array}{c}
q \\
j
\end{array}\right)=2^{q}
$$

and the proof is completed.

Combining (4.8), (4.10), and (4.11), we have

$$
\max _{n}\left|\triangle Q\left(e^{-i 2 \pi n / N}\right) / Q\left(e^{-i 2 \pi n / N}\right)\right|<\frac{2^{q+1}(q+1)}{\beta \delta} \max _{N \leq n \leq N+q}\left|t_{n}\right| .
$$

Since $\left|r_{i}\right|<1,1 \leq i \leq q, t_{n}$ is monotonically decreasing and

$$
\max _{N \leq n \leq N+q}\left|t_{n}\right|=\left|t_{N}\right|
$$

for sufficiently large $N$. Thus

$$
\max _{n}\left|\triangle Q\left(e^{-i 2 \pi n / N}\right) / Q\left(e^{-i 2 \pi n / N}\right)\right|<\frac{2^{q+1}(q+1)\left|t_{N}\right|}{\beta \delta}=\epsilon_{K}
$$

By Lemma 6 , there are at least $N-2 \max (p, q)$ eigenvalues of $Q_{N}^{-1} \triangle Q_{N}$ with magnitude bounded by $\epsilon_{K}$ in (4.13). Since eigenvalues of $T_{N}^{-1} \triangle T_{N}$ are equivalent to those of $Q_{N}^{-1} \triangle Q_{N}$, there are at least $N-2 \max (p, q)$ eigenvalues of $T_{N}^{-1} \triangle T_{N}$ with magnitude bounded by $\epsilon_{K}$ as well. When $\epsilon_{K}$ is small enough, there are at least $N-2 \max (p, q)$ eigenvalues of $K_{i, N}^{-1} T_{N}, i=1,2,3,4$, clustered between $\left(1-\epsilon_{K}, 1+\epsilon_{K}\right)$ for sufficiently large $N$. We summarize the analysis in this section into the following theorem.

THEOREM 4. Let $T_{m}$ be a sequence of $m \times m$ symmetric Toeplitz matrices generated by $T(z)$ with $T_{+}\left(z^{-1}\right)$ given by (3.3), and let the corresponding generating sequence satisfy (2.4) and (2.5). For sufficiently large $N$, the spectra of the preconditioned Toeplitz matrices $K_{i, N}^{-1} T_{N}, i=1,2,3,4$, have the following two properties:

$\mathrm{P} 1$. The number of outliers is at most $2 \max (p, q)$.

P2. There are at least $N-2 \max (p, q)$ eigenvalues that lie between $\left(1-\epsilon_{K}, 1+\epsilon_{K}\right)$, where $\epsilon_{K}$ is given by (4.13).

5. Discussion on Strang's preconditioners. We adopt a procedure similar to that described in $\S \S 3$ and 4 to examine the spectral properties of $S_{N}^{-1} T_{N}$, where $S_{N}$ is Strang's preconditioner. Only the cases where $p \leq q$ and $N=2 M$ are discussed. Since the analysis for the cases where $p>q$ or $N$ is odd can be performed in a straightforward way, it is omitted to avoid unnecessary repetition.

Recall that Strang's preconditioner $S_{N}$ is obtained by preserving the central halfdiagonals of $T_{N}$ and using them to form a circulant matrix. That is, when $N=2 M$, $S_{N}$ is defined as a symmetric Toeplitz matrix with the first row

$$
S_{N}:\left[t_{0}, t_{1}, \cdots, t_{M-1}, t_{M}, t_{M-1}, \cdots, t_{1}\right] .
$$

Let us denote the difference between $S_{N}$ and $T_{N}$ by $\triangle S_{N}$, i.e., $\triangle S_{N}=S_{N}-T_{N}$. The number of outliers of $S_{N}^{-1} T_{N}$ is determined by the following lemma.

Lemma 8. Let $T_{N}$ be an $N \times N$ symmetric Toeplitz matrix generated by $T(z)$ with $T_{+}\left(z^{-1}\right)$ given by (3.3), and let the corresponding generating sequence satisfy (2.4) and (2.5). $T_{N}^{-1} \triangle S_{N}$ has asymptotically at most $2 \max (p, q)$ nonzero eigenvalues (outliers). 
Proof. The proof is similar to that of Lemma 2. We use

$$
\triangle E_{N}=\left[\begin{array}{cc}
0 & \triangle F_{M} \\
\triangle F_{M}^{T} & 0
\end{array}\right]
$$

to approximate $\triangle S_{N}$, where

$$
\Delta F_{M}=\left[\begin{array}{ccccccc}
t_{M} & t_{M-1} & t_{M-2} & \cdots & t_{3} & t_{2} & t_{1} \\
t_{M+1} & t_{M} & t_{M-1} & \cdots & \cdot & t_{3} & t_{2} \\
t_{M+2} & t_{M+1} & t_{M} & \cdots & \cdot & \cdot & t_{3} \\
\cdot & \cdot & \cdot & \cdots & \cdot & \cdot & \cdot \\
t_{N-3} & \cdot & \cdot & \cdots & t_{M} & t_{M-1} & t_{M-2} \\
t_{N-2} & t_{N-3} & \cdot & \cdots & t_{M+1} & t_{M} & t_{M-1} \\
t_{N-1} & t_{N-2} & t_{N-3} & \cdots & t_{M+2} & t_{M+1} & t_{M}
\end{array}\right]
$$

Since elements $t_{n}$ in $\triangle F_{M}$ satisfy the recursion described in Lemma 1 , there are at most $\max (p, q)$ independent rows in $\triangle F_{M}$. Therefore, the rank of $\triangle E_{N}$ is at most $2 \max (p, q)$. Let us define $\triangle P_{N}=\triangle E_{N}-\triangle S_{N}$. Then we find that

$$
\triangle P_{N}=\left[\begin{array}{cc}
0 & \Delta G_{M} \\
\triangle G_{M}^{T} & 0
\end{array}\right]
$$

where $\triangle G_{M}$ is an $M \times M$ symmetric Toeplitz matrix with the first row

$$
\triangle G_{M}: \quad\left[t_{M}, t_{M+1}, t_{M+2}, \cdots, t_{N-3}, t_{N-2}, t_{N-1}\right] .
$$

It is easy to verify that, for sufficiently large $N$, the $l_{1}$ and $l_{\infty}$ norms of $\triangle P_{N}$ are both less than

$$
\tau_{S}=2 \sum_{n=M}^{N-1}\left|t_{n}\right|
$$

Consequently, we have

$$
\left\|\triangle P_{N}\right\|_{2} \leq\left(\left\|\triangle P_{N}\right\|_{1}\left\|\triangle P_{N}\right\|_{\infty}\right)^{1 / 2} \leq \tau_{S}
$$

Since $\tau_{S}$ goes to zero as $M$ goes to infinity due to (2.4), and since the eigenvalues of $T_{N}^{-1}$ are bounded due to (2.5), the spectra of $T_{N}^{-1} \triangle S_{N}$ and $T_{N}^{-1} \triangle E_{N}$ are asymptotically equivalent. It follows that both $T_{N}^{-1} \triangle E_{N}$ and $T_{N}^{-1} \triangle S_{N}$ have at most $2 \max (p, q)$ nonzero eigenvalues, asymptotically.

The matrix $\triangle S_{N}$ can be expressed as $\triangle S_{N}=\triangle S_{1, N}-\triangle S_{2, N}$, where

$$
\triangle S_{1, N}=\left[\begin{array}{cc}
0 & F_{1, M} \\
F_{1, M}^{T} & 0
\end{array}\right] \quad \text { and } \quad \Delta S_{2, N}=\left[\begin{array}{cc}
0 & F_{2, M} \\
F_{2, M}^{T} & 0
\end{array}\right]
$$

and where $F_{1, M}$ and $F_{2, M}$ are $M \times M$ upper triangular Toeplitz matrices with the following first rows:

$$
\begin{aligned}
& F_{1, M}:\left[t_{M}, t_{M-1}, t_{M-2}, \cdots, t_{2}, t_{1}\right] \\
& F_{2, M}:\left[t_{M}, t_{M+1}, t_{M+2}, \cdots, t_{N-2}, t_{N-1}\right] .
\end{aligned}
$$


We use $t_{n}$, which satisfies (3.6), to construct two new sequences:

$$
\begin{aligned}
& \tilde{s}_{1, n}= \begin{cases}0, & 0 \leq n \leq M-1, \\
t_{N-n}, & M \leq n \leq M+q-1, \\
-\left(\sum_{k=1}^{q} b_{q-k} \tilde{t}_{n-k}\right) / b_{q}, & M+q \leq n,\end{cases} \\
& \tilde{s}_{2, n}= \begin{cases}0, & 0 \leq n \leq M-1, \\
t_{n}, & M \leq n,\end{cases}
\end{aligned}
$$

and associate $\tilde{s}_{1, n}$ and $\tilde{s}_{2, n}$ with two sequences of symmetric Toeplitz matrices $\tilde{S}_{1, m}$ and $\tilde{S}_{2, m}, m=1,2, \cdots$, whose generating functions are defined as

$$
\tilde{S}_{1}(z)=\tilde{S}_{1,+}\left(z^{-1}\right)+\tilde{S}_{1,+}(z), \quad \text { where } \quad \tilde{S}_{1,+}\left(z^{-1}\right)=\frac{\tilde{s}_{1,0}}{2}+\sum_{n=1}^{\infty} \tilde{s}_{1, n} z^{-n},
$$

and

$$
\tilde{S}_{2}(z)=\tilde{S}_{2,+}\left(z^{-1}\right)+\tilde{S}_{2,+}(z), \quad \text { where } \quad \tilde{S}_{2,+}\left(z^{-1}\right)=\frac{\tilde{s}_{2,0}}{2}+\sum_{n=1}^{\infty} \tilde{s}_{2, n} z^{-n}
$$

respectively. We can easily verify that for $N>2 \max (p, q)$,

$$
\tilde{S}_{1, N}=\triangle S_{1, N} \quad \text { and } \quad \tilde{S}_{2, N}=\triangle S_{2, N}
$$

Then, by using the same approach for proving Lemma 3, we obtain the following lemma.

LEMmA 9. If $T_{N}$ is generated by $T(z)$ with $T_{+}\left(z^{-1}\right)$ given by (3.3), then

$$
\tilde{S}_{1,+}\left(z^{-1}\right)=\frac{z^{-M} \tilde{C}\left(z^{-1}\right)}{D\left(z^{-1}\right)}=\frac{\tilde{c}_{0}+\tilde{c}_{1} z^{-1}+\cdots+\tilde{c}_{q} z^{-q}}{d_{0}+d_{1} z^{-1}+\cdots+d_{q} z^{-q}}
$$

and

$$
\tilde{S}_{2,+}\left(z^{-1}\right)=\frac{z^{-M} \tilde{A}\left(z^{-1}\right)}{B\left(z^{-1}\right)}=\frac{\tilde{a}_{0}+\tilde{a}_{1} z^{-1}+\cdots+\tilde{a}_{q} z^{-q}}{b_{0}+b_{1} z^{-1}+\cdots+b_{q} z^{-q}}
$$

where the coefficients $b_{i}$ and $d_{i}$ are given by (3.3) and (3.8), and

$\tilde{a}_{i}=\left\{\begin{array}{ll}\sum_{j=0}^{i} b_{j} t_{M+i-j}, & 0 \leq i \leq q, \\ 0, & q<i,\end{array} \tilde{c}_{i}= \begin{cases}\sum_{j=0}^{i} d_{j} \tilde{s}_{1, M+i-j}, & 0 \leq i \leq q, \\ 0, & q<i,\end{cases}\right.$

with $\tilde{s}_{1, n}$ given by (5.1).

Thus $\triangle S_{N}$ can be decomposed into

$$
\triangle S_{N}=\triangle S_{1, N}-\triangle S_{2, N}=L_{\tilde{c}} L_{d}^{-1}+U_{\tilde{c}} U_{d}^{-1}-L_{\tilde{a}} L_{b}^{-1}-U_{\tilde{a}} U_{b}^{-1},
$$

where $L_{\tilde{c}}$ (or $U_{\tilde{c}}$ ) is an $N \times N$ lower (or upper) triangular Toeplitz matrix with the first $N$ coefficients of $z^{-M} \tilde{C}\left(z^{-1}\right)$ as its first column (or row), and matrices $L_{\tilde{a}}, L_{b}$, and $L_{d}$ (or $U_{\tilde{a}}, U_{b}$, and $U_{d}$ ) are similarly defined with respect to $z^{-M} \tilde{A}\left(z^{-1}\right), B\left(z^{-1}\right)$, and $D\left(z^{-1}\right)$, respectively.

By using the decomposition formulas (3.5) and (5.3), we transform the generalized eigenvalue problem

$$
\triangle S_{N} \mathbf{x}=\lambda T_{N} \mathbf{x}
$$


into another generalized eigenvalue problem

$$
\triangle Q_{S, N} \mathbf{y}=\lambda Q_{N} \mathbf{y}
$$

where

$$
\begin{aligned}
Q_{N} & =L_{b} T_{N} U_{b}=L_{a} U_{b}+L_{b} U_{a} \\
\triangle Q_{S, N} & =L_{b} \triangle S_{N} U_{b}=\left(L_{b} L_{\tilde{c}} L_{d}^{-1} U_{b}+L_{b} U_{\tilde{c}} U_{d}^{-1} U_{b}\right)-\left(L_{\tilde{a}} U_{b}+L_{b} U_{\tilde{a}}\right) .
\end{aligned}
$$

The systems (5.4) and (5.5) have the same eigenvalues and their eigenvectors are related via $\mathbf{x}=U_{b} \mathbf{y}$. The matrix $\Delta Q_{S, N}$ is a nearly banded Toeplitz matrix characterized by the following lemma.

LEMMA 10. Let $T_{m}$ be a sequence of $m \times m$ symmetric Toeplitz matrices generated by $T(z)$ with $T_{+}\left(z^{-1}\right)$ given by (3.3), and the corresponding generating sequence satisfies (2.4) and (2.5). The southeast $(N-\max (p, q)) \times(N-\max (p, q))$ block of $\triangle Q_{S, N}$ is a symmetric banded Toeplitz matrix with the generating function

$$
\triangle Q_{S}(z)=B\left(z^{-1}\right) \tilde{S}(z) B(z)=\triangle Q_{S, 1}(z)-\triangle Q_{S, 2}(z),
$$

where

$$
\begin{aligned}
& \triangle Q_{S, 1}(z)=b_{q} z^{-(M-q)} B\left(z^{-1}\right) \tilde{C}\left(z^{-1}\right)+b_{q} z^{M-q} B(z) \tilde{C}(z) \\
& \triangle Q_{S, 2}(z)=z^{-M} \tilde{A}\left(z^{-1}\right) B(z)+z^{M} B\left(z^{-1}\right) \tilde{A}(z)
\end{aligned}
$$

Since the generating sequence $t_{n}$ of $T_{N}$ satisfies conditions (2.4) and (2.5), we can use arguments given in the previous section and obtain

$$
\left|\frac{\triangle Q_{S, 1}\left(e^{-i \theta}\right)}{Q\left(e^{-i \theta}\right)}\right| \leq \frac{2^{q+1}(q+1)\left|t_{M}\right|}{\beta \delta}=\epsilon^{\prime}
$$

and

$$
\left|\frac{\triangle Q_{S, 2}\left(e^{-i \theta}\right)}{Q\left(e^{-i \theta}\right)}\right| \leq \frac{2^{q+1}(q+1)\left|t_{M}\right|}{\beta \delta}=\epsilon^{\prime}
$$

for arbitrary $\theta$. By using arguments similar to those in Lemma 6, it can be derived that $T_{N}^{-1} \triangle S_{N}$ has at least $N-2 \max (p, q)$ eigenvalues bounded by

$$
\epsilon_{S}=2 \epsilon^{\prime}=\frac{2^{q+2}(q+1)\left|t_{N / 2}\right|}{\beta \delta}
$$

for sufficiently large $N$. The analysis in this section is concluded by the following theorem.

THEOREM 5. Let $T_{m}$ be a sequence of $m \times m$ symmetric Toeplitz matrices generated by $T(z)$ with $T_{+}\left(z^{-1}\right)$ given by (3.3), and the corresponding generating sequence satisfies (2.4) and (2.5). For sufficiently large $N$, the spectrum of the preconditioned Toeplitz matrix $S_{N}^{-1} T_{N}$ has the following two properties:

$\mathrm{P} 1$. The number of outliers is at most $2 \max (p, q)$.

P2. There are at least $N-2 \max (p, q)$ eigenvalues that lie between $\left(1-\epsilon_{S}, 1+\epsilon_{S}\right)$, where $\epsilon_{S}$ is given by (5.7).

Let us compare the preconditioners $K_{i, N}$ and $S_{N}$. From Theorems 4 and 5, the spectra of $K_{i, N}^{-1} T_{N}$ and $S_{N}^{-1} T_{N}$ have the same number of outliers, and the other 
eigenvalues are clustered around 1 within radii $\epsilon_{K}$ and $\epsilon_{S}$ given by (4.13) and (5.7), respectively. It is clear that the parameters $q, \beta$, and $\delta$ are independent of the problem size $N$, and that the terms $\left|t_{N}\right|$ and $\left|t_{N / 2}\right|$ determine the convergence rate of the PCG method. For sufficiently large $N$, we have $O\left(\epsilon_{K}\right)=O\left(\epsilon_{S}^{2}\right)$. This implies that, after the first several iterations which eliminate the effects of the outliers, the residual reduced by one iteration of the PCG method with preconditioners $K_{i, N}$ is about the same as that reduced by two iterations of the PCG method with preconditioner $S_{N}$. This has been confirmed by numerical experiments reported in [15].

6. The special case with geometric generating sequences. It has been observed from numerical experiments [15], [21], that the eigenvalues of $K_{1, N}^{-1} T_{N}$ and $S_{N}^{-1} T_{N}$ with $T_{N}$ generated by the geometric sequence $t_{n}=t^{n},|t|<1$, are very regular. The observations are summarized as follows.

R1. The eigenvalues of $K_{1, N}^{-1} T_{N}$ are $(1+t)^{-1},(1-t)^{-1}$, and $\left(1-t^{N}\right)^{-1}$ with multiplicities 1,1 , and $N-2$, respectively.

R2. When $N$ is even $(N=2 M)$, the eigenvalues of $S_{N}^{-1} T_{N}$ are $(1+t)^{-1},(1-t)^{-1}$, $1,\left(1+t^{M}\right)^{-1}$, and $\left(1-t^{M}\right)^{-1}$ with multiplicities $1,1,2, M-2$, and $M-2$, respectively.

In this section, we provide an analytical approach to explain these two regularities.

First, we examine the preconditioner $K_{1, N}$. For the generating sequence $t_{n}=t^{n}$, its generating function is

$$
T(z)=T_{+}\left(z^{-1}\right)+T_{+}(z), \quad \text { where } T_{+}\left(z^{-1}\right)=\frac{A\left(z^{-1}\right)}{B\left(z^{-1}\right)}=\frac{0.5+0.5 t z^{-1}}{1-t z^{-1}}
$$

so that the order $(p, q)$ of $T_{+}\left(z^{-1}\right)$ is $(1,1)$. From Lemma 3 , we obtain

$$
\tilde{T}_{+}\left(z^{-1}\right)=\frac{C\left(z^{-1}\right)}{D\left(z^{-1}\right)}=\frac{t^{N}\left(0.5+0.5 t^{-1} z^{-1}\right)}{1-t^{-1} z^{-1}},
$$

which is related to $\Delta T_{N}=K_{1, N}-T_{N}$. By using (4.3) and (4.4), we have

$$
Q(z)=A\left(z^{-1}\right) B(z)+B\left(z^{-1}\right) A(z)=1-t^{2}
$$

and

$$
\triangle Q(z)=-t\left[z B\left(z^{-1}\right) C\left(z^{-1}\right)+z^{-1} B(z) C(z)\right]=-t^{N}\left(1-t^{2}\right) .
$$

Note that $q=1$ and $b_{q}=-t$ are used in deriving (6.2). Due to (6.1) and (6.2), the southeast $(N-1) \times(N-1)$ blocks of $Q_{N}$ and $\triangle Q_{N}$ are identity matrices multiplied by the constants $1-t^{2}$ and $-t^{N}\left(1-t^{2}\right)$, respectively. Consider the following linear combination of $Q_{N}$ and $\triangle Q_{N}$ :

$$
V_{N}=\triangle Q_{N}+t^{N} Q_{N}
$$

It is clear that the southeast $(N-1) \times(N-1)$ block of $V_{N}$ is a zero matrix. Since the first two columns are linearly independent, and any two columns of the last $N-1$ columns of $V_{N}$ are linearly dependent, $V_{N}$ has a null space of dimension $N-2$. This implies that $Q_{N}^{-1} \triangle Q_{N}$, or equivalently, $T_{N}^{-1} \triangle T_{N}$, has the eigenvalue $-t^{N}$ with multiplicity $N-2$. Therefore, $\left(T_{N}+\Delta T_{N}\right)^{-1} T_{N}=K_{1, N}^{-1} T_{N}$ has the eigenvalue $\left(1-t^{N}\right)^{-1}$ with multiplicity $N-2$.

To determine the remaining two eigenvalues, i.e., the outliers, we use the technique described in [4] to transform the problem $\triangle T_{N} \mathbf{x}=\lambda T_{N} \mathbf{x}$ to another equivalent 
problem. Consider the case with even $N(N=2 M)$. Since $\triangle T_{N}$ and $T_{N}$ are both symmetric Toeplitz matrices, they can be expressed in the following block matrix form:

$$
\Delta T_{N}=\left[\begin{array}{cc}
\triangle T_{1, M} & \triangle T_{2, M}^{T} \\
\triangle T_{2, M} & \triangle T_{1, M}
\end{array}\right] \quad \text { and } \quad T_{N}=\left[\begin{array}{cc}
T_{1, M} & T_{2, M}^{T} \\
T_{2, M} & T_{1, M}
\end{array}\right]
$$

Let $W_{N}$ be the orthonormal matrix

$$
W_{N}=\frac{1}{\sqrt{2}}\left[\begin{array}{cc}
I_{M} & I_{M} \\
-J_{M} & J_{M}
\end{array}\right]
$$

where $I_{M}$ and $J_{M}$ are $M \times M$ identity and symmetric elementary matrices, respectively. By using the transformation

$$
W_{N}^{-1} \triangle T_{N} W_{N} \mathbf{y}=\lambda W_{N}^{-1} T_{N} W_{N} \mathbf{y}
$$

we obtain two decoupled subproblems,

$$
\begin{aligned}
& \left(\triangle T_{1, M}-J_{M} \triangle T_{2, M}\right) \mathbf{y}_{--}=\lambda_{-}\left(T_{1, M}-J_{M} T_{2, M}\right) \mathbf{y}_{-}, \\
& \left(\triangle T_{1, M}+J_{M} \triangle T_{2, M}\right) \mathbf{y}_{+}=\lambda_{+}\left(T_{1, M}+J_{M} T_{2, M}\right) \mathbf{y}_{+},
\end{aligned}
$$

where $\lambda_{-}$and $\lambda_{+}$are also eigenvalues of the original problem $\Delta T_{N} \mathbf{x}=\lambda T_{N} \mathbf{x}$. Since the first rows of matrices on both sides of (6.3) are proportional by a constant $-t$, $\lambda_{-}=-t$ with $\mathbf{y}_{-}=\mathbf{e}_{1}$ (the unit vector with 1 at the first element) satisfies (6.3). Similarly, we can argue that $\lambda_{+}=t$ with $\mathbf{y}_{+}=\mathbf{e}_{1}$ is an eigenvalue-eigenvector pair for (6.4). Thus $1 /(1-t)$ and $1 /(1+t)$ are two outliers of $\left(T_{N}+\triangle T_{N}\right)^{-1} T_{N}=K_{1, N}^{-1} T_{N}$. When $N$ is odd, the same result can be derived with a slightly modified $W_{N}$ given in [4].

By using the relationship among preconditioners $K_{i, N}, i=1,2,3,4$, we can determine all eigenvalues of $K_{i, N}^{-1} T_{N}$. They all have three distinct eigenvalues (two outliers and $N-2$ clustered eigenvalues) summarized in Table 1.

TABLE 1

Eigenvalues of $K_{i, N}^{-1} T_{N}$.

\begin{tabular}{|c||c|c|c|c|}
\hline & $K_{1, N}^{-1} T_{N}$ & $K_{2, N}^{-1} T_{N}$ & $K_{3, N}^{-1} T_{N}$ & $K_{4, N}^{-1} T_{N}$ \\
\hline \hline$\lambda_{1}$ & $(1+t)^{-1}$ & $(1+t)^{-1}$ & $(1+t)^{-1}$ & $(1-t)^{-1}$ \\
\hline$\lambda_{2}$ & $(1-t)^{-1}$ & $(1-t)^{-1}$ & $\left(1+t^{N}\right)^{-1}$ & $\left(1+t^{N}\right)^{-1}$ \\
\hline$\lambda_{3}$ & $\left(1-t^{N}\right)^{-1}$ & $\left(1+t^{N}\right)^{-1}$ & $\left(1-t^{N}\right)^{-1}$ & $\left(1-t^{N}\right)^{-1}$ \\
\hline
\end{tabular}

Next, we examine Strang's preconditioner $S_{N}$ with even $N$. When $N=2 M$, the two central rows of $S_{N}-T_{N}$ are zeros. This implies that $S_{N}^{-1} T_{N}$ has the eigenvalue 1 with multiplicity 2 . By using (5.2a) and (5.2b), we have

$$
\begin{aligned}
& \tilde{S}_{1,+}\left(z^{-1}\right)=\frac{z^{-M} \tilde{C}\left(z^{-1}\right)}{D\left(z^{-1}\right)}=\frac{z^{-M} t^{M}}{1-t^{-1} z^{-1}}, \\
& \tilde{S}_{2,+}\left(z^{-1}\right)=\frac{z^{-M} \tilde{A}\left(z^{-1}\right)}{B\left(z^{-1}\right)}=\frac{z^{-M} t^{M}}{1-t z^{-1}}
\end{aligned}
$$


respectively. By substituting $\tilde{A}\left(z^{-1}\right), B\left(z^{-1}\right), \tilde{C}\left(z^{-1}\right)$, and $D\left(z^{-1}\right)$ into (5.6) and using (6.1), we obtain

$$
\triangle Q_{S}(z)=-t^{M}\left(z^{-M}+z^{M}\right)\left(1-t^{2}\right) .
$$

Then, the nonzero elements of $\triangle \mathcal{Q}_{S, N-1}$, which is the southeast $(N-1) \times(N-1)$ block of $\triangle Q_{S, N}$, only occur along the $\pm M$ th diagonals and take the same value $-t^{M}\left(1-t^{2}\right)$. Consider the linear combination of $\triangle Q_{S, N}$ and $Q_{N}$,

$$
V_{1, N}=\triangle Q_{S, N}+t^{M} Q_{N}
$$

By adding the $k+1$ th column to the $M+k+1$ th column of $V_{1, N}$, for $k=1,2, \cdots(M-$ $1)$, we find that the southeast $(N-1) \times(M-1)$ block of the resulting matrix is the zero matrix. Consequently, $V_{1, N}$ has a null space of dimension $M-2$ and $Q_{N}^{-1} \triangle Q_{S, N}$ has the eigenvalue $-t^{M}$ with multiplicity $M-2$. Similarly, we can show that

$$
V_{2, N}=\triangle Q_{S, N}-t^{M} Q_{N}
$$

has a null space of dimension $M-2$ by subtracting the $k+1$ column from the $M+k+1$ th column of $V_{2, N}$, for $k=1,2, \cdots(M-1)$. Therefore, $Q_{N}^{-1} \triangle Q_{S, N}$ has the eigenvalue $t^{M}$ with the same multiplicity $M-2$. As a consequence, $S_{N}^{-1} T_{N}$ has the eigenvalues $\left(1+t^{M}\right)^{-1}$ and $\left(1-t^{M}\right)^{-1}$ with multiplicity $M-2$.

To determine the remaining two eigenvalues of $S_{N}^{-1} T_{N}$, we use the same transformation discussed earlier and consider the eigenvalues of the following two subproblems:

$$
\begin{aligned}
& \left(T_{1, M}-J_{M} T_{2, M}\right) \mathbf{y}_{-}=\lambda_{-}\left(S_{1, M}-J_{M} S_{2, M}\right) \mathbf{y}_{-} \\
& \left(T_{1, M}+J_{M} T_{2, M}\right) \mathbf{y}_{+}=\lambda_{+}\left(S_{1, M}+J_{N} S_{2, M}\right) \mathbf{y}_{+}
\end{aligned}
$$

where $S_{1, M}$ and $S_{2, M}$ are the northwest and southwest $M \times M$ blocks of $S_{N}$, respectively. Since the first rows of matrices on both sides of (6.5) are proportional by a constant $1-t, \lambda_{-}=1 /(1-t)$ with $\mathbf{y}_{-}=\mathbf{e}_{1}$ satisfies (6.5). Similarly, $\lambda_{+}=1 /(1+t)$ with $\mathbf{y}_{+}=\mathbf{e}_{1}$ satisfies (6.6).

7. Conclusion. In this paper, we have proved the spectral properties of the preconditioned rational Toeplitz matrices $P_{N}^{-1} T_{N}$ with the preconditioner $S_{N}$ proposed by Strang [19] and the preconditioners $K_{i, N}$ proposed by the authors [15]. The eigenvalues of $P_{N}^{-1} T_{N}$ are classified into two classes, i.e., the outliers and the clustered eigenvalues. The number of outliers depends on the order of the rational generating function. The clustered eigenvalues are confined in the interval $(1-\epsilon, 1+\epsilon)$ with the radii $\epsilon_{K}=O\left(\left|t_{N}\right|\right)$ and $\epsilon_{S}=O\left(\left|t_{N / 2}\right|\right)$ for $K_{i, N}^{-1} T_{N}$ and $S_{N}^{-1} T_{N}$, respectively. When the symmetric Toeplitz matrix $T_{N}$ is generated by the geometric sequence $t^{n}$ with $|t|<1$, the precise eigenvalue distributions of $K_{i, N}^{-1} T_{N}$ and $S_{2 M}^{-1} T_{2 M}$ have been determined analytically. Since the eigenvalues of $K_{i, N}^{-1} T_{N}$ are more closely clustered than those of $S_{N}^{-1} T_{N}$, preconditioners $K_{i, N}$ are more efficient for solving rational Toeplitz systems.

\section{REFERENCES}

[1] G. S. Ammar AND W. B. GragG, Superfast solution of real positive definite Toeplitz systems, SIAM J. Matrix Anal. Appl., 9 (1988), pp. 61-76. 
[2] R. R. Bitmead AND B. D. Anderson, Asymptotically fast solution of Toeplitz and related systems of equations, Linear Algebra Appl., 34 (1980), pp. 103-116.

[3] R. P. Brent, F. G. Gustavson, And D. Y. Yun, Fast solution of Toeplitz systems of equations and computations of Padé approximations, J. Algorithms, 1 (1980), pp. 259295.

[4] A. CANTONi AND P. BUtler, Eigenvalues and eigenvectors of symmetric centrosymmetric matrices, Linear Algebra Appl., 13 (1976), pp. 275-288.

[5] R. H. ChAN, Circulant preconditioners for Hermitian Toeplitz system, SIAM J. Matrix Anal. Appl., 10 (1989), pp. 542-550.

[6] - The spectrum of a family of circulant preconditioned Toeplitz systems, SIAM J. Numer. Anal., 26 (1989), pp. 503-506.

[7] R. H. ChAN AND G. STRANG, Toeplitz equations by conjugate gradients with circulant preconditioner, SIAM J. Sci. Statist. Comput., 10 (1989), pp. 104-119.

[8] T. F. Chan, An optimal circulant preconditioner for Toeplitz systems, SIAM J. Sci. Statist. Comput., 9 (1988), pp. 766-771.

[9] P. Davis, Circulant Matrices, John Wiley, New York, 1979.

[10] P. Delsarte AND Y. V. Genin, The split Levinson algorithm, IEEE Trans. Acoust. Speech Signal Process., ASSP-34 (1986), pp. 470-478.

[11] B. W. Dickinson, Efficient solution of linear equations with banded Toeplitz matrices, IEEE Trans. Acoust. Speech Signal Process., ASSP-27 (1979), pp. 421-422.

[12] - Solution of linear equations with rational Toeplitz matrices, Math. Comp., 34 (1980), pp. 227-233.

[13] F. D. Hoog, A new algorithm for solving Toeplitz systems of equations, Linear Algebra Appl., 88/89 (1987), pp. 123-138.

[14] T. HUCKLE, Circulant and skew-circulant matrices for solving Toeplitz matrices problems, in Copper Mountain Conference on Iterative Methods, Copper Mountain, CO, 1990; SIAM J. Matrix Anal. Appl., 13 (1992), pp. 767-777.

[15] T. K. KU AND C. J. KUO, Design and analysis of Toeplitz preconditioners, IEEE Trans. Signal Process., 40 (1992), pp. 129-141.

[16] N. LEVInSON, The Wiener RMS error criterion in filter design and prediction, J. Math. Phys., 25 (1947), pp. 261-278.

[17] H. S. MAlvar, Fast computation of the discrete Cosine transform and the discrete Hartley transform, IEEE Trans. Acoust. Speech Signal Process., ASSP-35 (1987), pp. 1484-1485.

[18] H. V. Sorensen, D. L. Jones, M. T. Heideman, And C. S. Burrus, Real-value fast Fourier transform algorithms, IEEE Trans. Acoust. Speech Signal Process., ASSP-35 (1987), pp. 849-863.

[19] G. Strang, A proposal for Toeplitz matrix calculations, Stud. Appl. Math., 74 (1986), pp. 171176.

[20] - Linear Algebra and Its Applications, Harcourt Brace Jonanovich, Inc., Third Edition, Orlando, FL, 1988.

[21] G. Strang And A. Edelman, The Toeplitz-circulant eigenvalue problem $A x=\lambda C x$, in Oakland Conference on PDE's, L. Bragg and J. Dettman, eds., Longmans, London, 1987.

[22] L. N. Trefethen, Rational approximation on the unit disk, Numer. Math., 37 (1981), pp. 297320.

[23] - Approximation theory and numerical linear algebra, in Algorithms for Approximation II, M. Cox and J. C. Mason, eds., Chapman, London, New York, 1988.

[24] W. F. TRENCH, Solution of systems with Toeplitz matrices generated by rational functions, Linear Algebra Appl., 74 (1986), pp. 191-211.

[25] - Toeplitz systems associated with the product of a formal Laurent series and a Laurent polynomial, SIAM J. Matrix Anal. Appl., 9 (1988), pp. 181-193.

[26] J. H. Wilkinson, The Algebraic Eigenvalue Problem, Oxford University Press, New York, 1965.

[27] P. YIP AND K. R. RAO, A fast computational algorithm for the discrete Sine transform, IEEE Trans. Comm., COM-28 (1980), pp. 304-307. 